\title{
Towards an Understanding of the Effect of Night Vision Display Imagery on Scene Recognition
}

\author{
Geoffrey W. Stuart ${ }^{*}$ and Philip K. Hughes
}

Air Operations Division, Defence Science and Technology Organisation, 506 Lorimer St, Fishermans Bend, VIC 3207, Australia

\begin{abstract}
An experimental study was carried out to evaluate the combined effect of the absence of colour and the alteration of surface intensities on the recognition of complex scenes. This approach was motivated by the properties of the monochrome imagery of night vision goggles (NVGs), which are a commonly used form of night vision display. Observers were presented with pairs of aerial views of simulated urban scenes (from 400 or $700 \mathrm{ft}$ ), taken from viewing angles that differed by $30 \mathrm{deg}$ to ensure that participants could not rely on a viewpoint-dependent cognitive representation of the scene. The observer's task was to decide whether the two scenes were the same, apart from the rotated viewpoint. On catch trials, which were fewer in number, one of the scenes was also mirror reversed. These trials were included to prevent guessing or premature responses. On half the trials, one of the scenes was rendered to simulate the effects of night-vision imagery. The time taken for the observers to confirm the identity of the rotated scenes was measured. There was no effect of differing altitude. When both of the scenes were rendered as daylight imagery, the average time to achieve a match was $34.7 \mathrm{~s}$. When one of the scenes was rendered as NVD-style imagery, the matching time rose to $50.8 \mathrm{~s}$. This effect varied according to the complexity of the scene and the extent to which the scene contained salient coloured features. There were also pronounced differences between observers. These findings suggest that night vision displays may have adverse effects on scene recognition compared to viewing natural-coloured images of the same scenes.
\end{abstract}

\section{INTRODUCTION}

Night vision displays (NVDs) are commonly used in military aviation. Most NVDs in current use employ single sensors either in the visible and near infrared range, or in the mid-infrared range [1]. Examples of such devices are the night vision goggles (NVGs) worn by soldiers and pilots, which rely on the amplification of reflected light, and infrared (IR) displays that use the heat radiated from objects to produce an image of the environment. The tactical advantages of a night-flight capability are an obvious justification for the use of NVDs. Nonetheless, these aids do not "turn night into day". With respect to the factors that potentially degrade visual performance, Rash, Verona and Crowley [2] and Hughes [3] identified loss of visual acuity, reduced contrast sensitivity, reduced field of view, impaired depth perception, loss of colour, and altered appearance of surface brightness as negative factors associated with NVDs.

The use of NVDs in helicopter flight has been found to greatly increase the risk of accident due to spatial disorientation. For example, Braithwaite, Douglass, Durnford and Lucas [4] reported that the rate of fatal accidents due to spatial disorientation was over five times higher when flying with NVDs. These findings indicate that NVD-assisted helicopter flight involves increased risk, a fact recognised by operating procedures imposing speed and height limitations for this kind of flight. Although fatal accidents represent the most extreme

*Address correspondence to this author at the Air Operations Division, Defence Science and Technology Organisation, 506 Lorimer St, Fishermans Bend, VIC 3207, Australia; E-mail: Geoff.Stuart@dsto.defence.gov.au outcome of NVD disorientation, the magnitude of the increased risk indicates that there may also be a greater likelihood of spatial disorientation from which the aircrew can recover, or instances where they simply end up becoming disorientated or lost temporarily. Such incidents are less likely to be reported and subjected to analysis, but may nevertheless have negative operational consequences. Indeed, anecdotal reports by flight crew suggest that geographical or man-made features may be sometimes difficult to recognise due to their unfamiliar appearance, increasing the risk of geographical disorientation.

There are two factors that may contribute to this difficulty. When viewing the world through an NVD, colour information that might be used to segment and otherwise organise the scene and to search for particular features and landmarks is absent. Most NVDs in current use employ single sensors in either the visible/near infrared range, or in the mid infrared range. The devices use monochrome displays, but the type of display differs markedly between devices. NVGs, which are based on image intensifiers, usually use green phosphors. NVGs may be worn directly, or the imagery may be projected onto a helmet-mounted display. Forward-looking infrared (FLIR) uses a grey-scale monochrome display, with an option for inverting intensities. FLIR imagery can be displayed on an instrument panel, a fixed head-up display, or on a helmet-mounted display.

Experimental studies of scene and object recognition have shown that colour information can aid both the rapid recognition of single objects that have prototypical colours, for example, a banana [5] and broad types of scene, such as deserts, forests and coast [6]). Goffeaux et al. [7] showed 
that when rapidly categorising this scene "gist" observers made the most rapid responses when the scene was diagnostically (naturally) coloured, were slower when the scene was rendered as a greyscale, and even slower when rendered in unnatural colours. This suggests that at least the intial perception of the scene is aided by natural colouration and may be impaired by loss of colour information, and in the case of NVDs, unnatural grey-scale rendering. However, there have been no studies to date that have examined the effects of loss of colour and altered reflectances on the detailed perception of complex scenes (i.e., configurations of landmarks) that is required for effective visually-based navigation. This is a much slower process that requires the acquisition of a viewpoint independent representation of the scene [8-10].

Although there has been a great deal of research on the land-based navigation abilities of individuals without specialised training, usually as pedestrians, the cognitive skills involved in flight navigation are less well understood. Wickens [11] has presented the most comprehensive analysis to date of the cognitive demands of airborne navigation. The concept of the frame of reference occupies a central position in this framework. When using a map or other navigational aid, the navigator must convert an egocentric frame of reference, that is, the forward field of view out of the aircraft, which is determined by the current altitude and heading, to an exocentric frame of reference, most commonly represented by a North-up, plan-view map. A number of researchers have studied this process, and it has been found that the time taken to make a navigational decision depends on the difference in angular and elevation viewpoints between the map and the outside world [12-15]. In the case of plan-view maps, it has been shown that a prototypic elevation angle of about $30 \mathrm{deg}$ is used to generate the internal 3-D representation for comparison with the outside world [12]. Departures from this elevation angle seem to necessitate additional mental rotation with concomitant increases in decision time.

Fig. (1) provides a schematic representation of stages in the recognition of a visual scene, presented as daylight or NVD imagery. The observer must first derive an impression of the scene to be recognised from some source of visual information. This may be in the form of a visual aid such as a map or photograph, or may be derived from longer-term memory, acquired during reconnaissance, or during mission rehearsal in a simulator. Due to limitations in workingmemory capacity (even when using a visual aid) certain key features must be extracted and placed in short-term visual memory. This abstract representation must then be mentally rotated and matched to the external scene. If a match cannot be definitely confirmed or rejected, the observer may need to check the visual aid (or their long-term memory) again, and extract new features and their spatial configuration.

When navigating with the aid of an NVD, the final stage of this matching process requires the observer to generate an internal, viewpoint-independent representation of what the scene represented on the map may look like. This internal representation may reflect the usual appearance of the landmarks and geographical features, including colours and luminous reflectances. This may present a problem when the outside world is seen in the unfamiliar mode generated by an NVD. Specifically, the navigator must make a decision about whether the view of the outside world corresponds to that represented on the map, in the face of the additional cognitive demand of interpreting the NVD imagery of the outside world.

The component of the complex navigation task that involves mental rotation and scene matching is the subject of the present investigation. The cognitive operations involved in converting a map representation (paper or electronic) to an internal mental representation of the real world have already

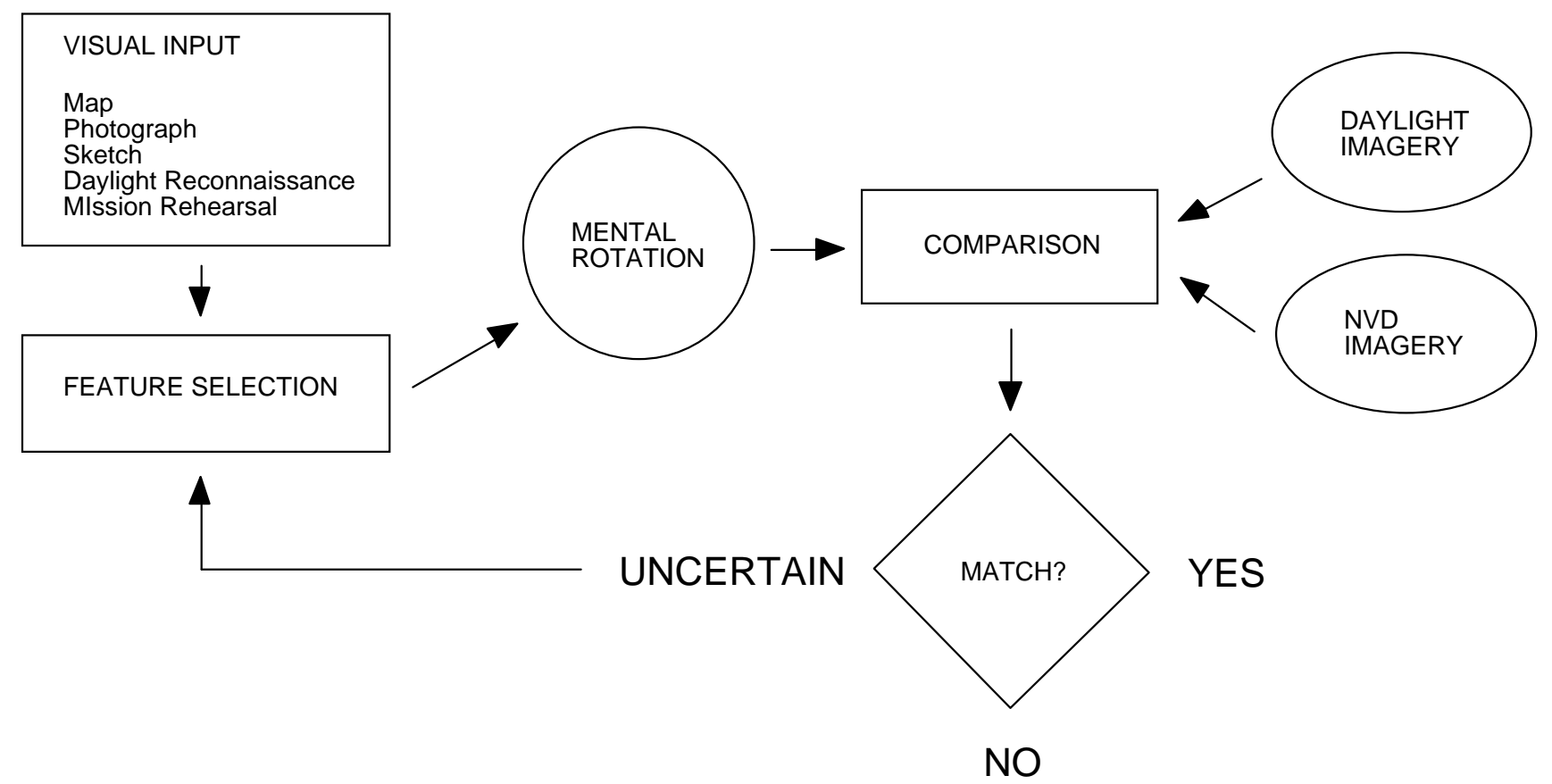

Fig. (1). A heuristic model of the cognitive operations underlying scene recognition. See text for further explanation. 
been elucidated by others [12-15]. However, the exact nature of this mental representation is not clear. In particular, the role that colour information plays in this process is unknown. If scene recognition relies heavily on the spatial layout of key features, and those features are recognised on the basis of their size and shape alone, colour information and other surface properties such as luminance and texture may be irrelevant to the task. On the other hand, it may be conjectured that the ability of humans to segment scenery in the absence of colour relies on visual features such as texture that depend on the high resolution of the visual system. It is possible that when this high-spatial frequency information is absent, as in NVDs, colour may provide valuable compensatory information. In this study, the central focus will be on the mental rotation of one scene into correspondence with another for the purpose of scene recognition. Of particular interest is the effect that visual losses similar to those produced by NVDs will have on this process.

\section{METHOD}

\subsection{Participants}

The participants were 16 healthy volunteers aged from 24 to 42 years (median age 27.8). Of these, 3 were female. All had normal or corrected vision, and were tertiary-educated scientists. None had any previous operational experience with NVDs. One was qualified to fly light aircraft.

\subsection{Stimuli}

The experimental stimuli were static aerial views of cities acquired from Microsoft Flight Simulator $98^{\circ}$. This program has a facility that allows the user to proceed to a given geographical coordinate, to an accuracy of $1 / 100$ th of a minute of latitude and longitude, and at a specific altitude and heading. Calculations took into account the magnetic declination at each location, given that headings are relative to magnetic north. The distance from each simulated city was set so that the tallest buildings took up around the same vertical proportion of the image (approximately $60 \%$ ), and the pitch was likewise set to place the horizon line in a consistent position, which varied according to altitude. In this manner, four still snapshots of 16 cities were taken, from two heights (400 and $700 \mathrm{ft}$ ), and from headings approximately $30 \mathrm{deg}$ apart. Example images of the 16 locations, in daylight imagery, are shown in Fig. (2). For this purpose, all cockpit imagery was not displayed using an option in the program. The final cropped images were 640 pixels wide and 240 pixels high.

In order to simulate visual losses similar to those associated with NVDs, these four images of each city were subjected to the following manipulations. First, each image was transformed to a green monochrome image by replacing the red and blue values of each 24-bit pixel triplet with zeroes. The image was then reversed in contrast, and a saturating piecewise linear transform applied to remove contrast between relatively bright areas of the image (all values above a set threshold were set to the maximum value). These manipulations were used to simulate the fact that the sky is bright during the day, but dark in IR images, and similarly, windows emit heat at night, but appear dark during the day. The saturation effect emulated the "flaring" of bright light and heat sources due to the high sensitivity of the sensors. Examples are shown in Fig. (2B, C). This was not a true night-vision rendition of the daylight imagery, which would require knowledge about specific reflectances of the surfaces in the images at the wavelengths to which specific sensors are sensitive. However, it incorporates two important distortions associated with such devices that may affect scene recognition, namely, loss of colour and altered surface luminance.

\subsection{Apparatus}

Images were presented on an IBM-compatible Pentium 100 personal computer, running MS DOS 6.22. Subjects were seated approximately $70 \mathrm{~cm}$ from a 15 inch monitor (Samsung 15Gle). The graphics mode was 24-bit colour at a vertical refresh rate of $60 \mathrm{~Hz}$. The target stimulus was always presented as daylight imagery at the top of the screen, and the comparison stimulus immediately below. The two images took up the display area of the screen, which was 640 by 480 pixels. The horizontal visual angle subtended by the images was $21 \mathrm{deg}$. Responses were collected using a game pad interfaced to the games port of the computer. A long period timer with a resolution of $1 / 18$ th of a second was used to time the participants' responses.

\subsection{Design}

The design of the experiment was as follows. There were 8 different experimental conditions in a $2 \times 2 \times 2$ design. The target image, which was always in colour, was taken from an altitude of either 400 or $700 \mathrm{ft}$. The comparison image (which was always from a viewpoint 30 deg different from the target scene, and presented below it on the screen) was also taken from either 400 (low) or $700 \mathrm{ft}$ (high). The purpose of these manipulations was to ensure that the participant had apprehended the three-dimensional spatial layout of the scene [10], an essential component of scene recognition for navigation. Thus there were four possible combinations involving altitude: low-low, low-high, highlow and high-high. For each of these four combinations, the comparison image was rendered in daylight or simulated night-vision display imagery. Thus the comparison image was always of the same city, but differed in a specified combination of viewpoint and type of rendering.

Interspersed with the experimental trials were "catch trials", which occurred with a 1-in-3 probability, and encompassed all types of experimental condition. On these trials, as well as being rotated $30 \mathrm{deg}$, the comparison image was reflected from left to right. This is a common device used in experimental studies of mental rotation [16]. The principal advantage is that there is good control for the individual features of the display, in this case, the number, style, and colour of buildings. In selecting the experimental stimuli, an effort was made to avoid including obvious clues to mirror rotation, so that the participants had to make a more global judgement of the configuration of buildings to decide whether the two scenes could be rotated into correspondence. Examples of stimulus pairs are shown in Fig. (3A-C). These increase in difficulty from the top to the bottom of the figure. Some are matches, and others are mirror-reversed catch trials. 

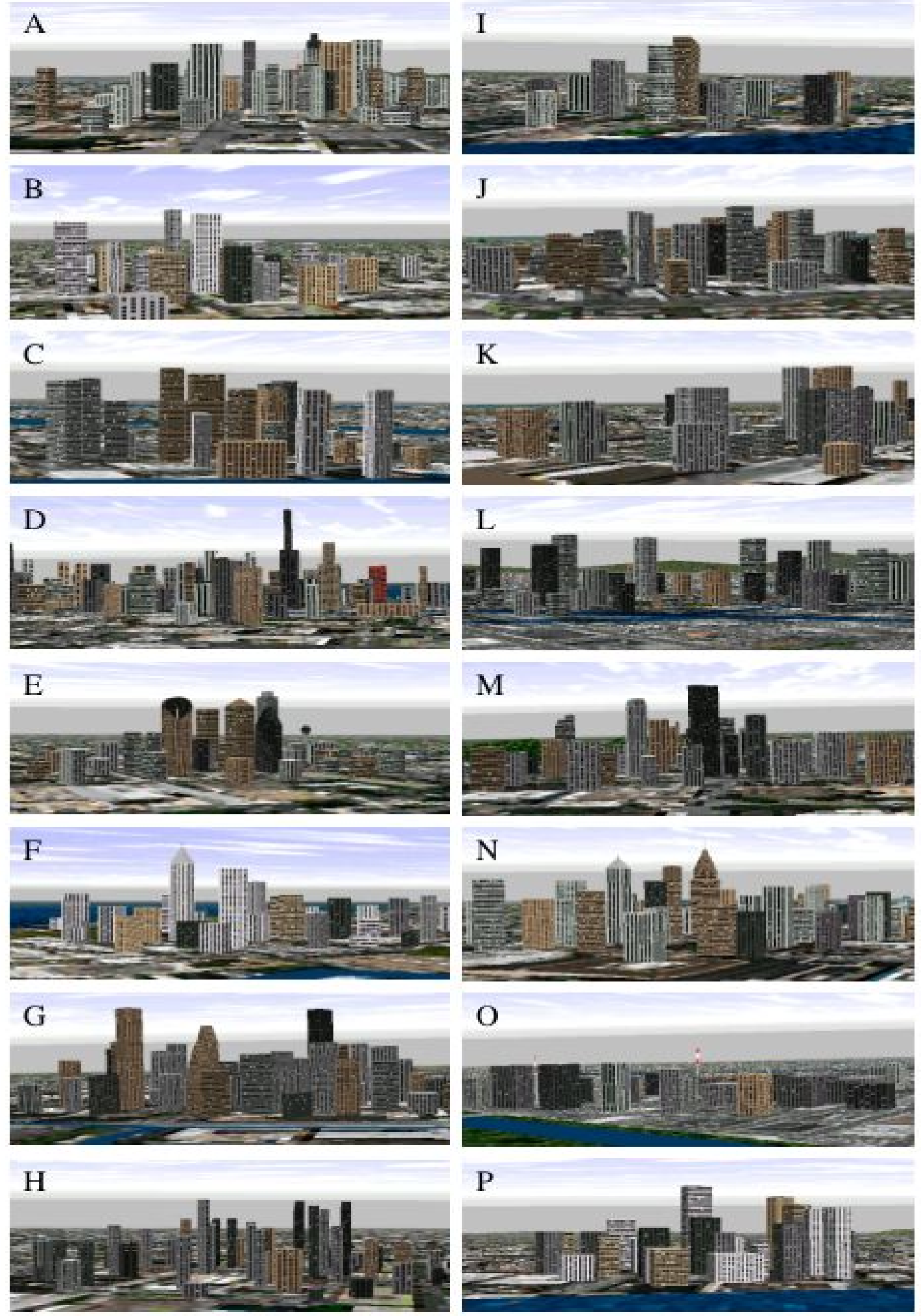

Fig. (2). Examples of the simulated urban scenes used in the experiment. All scenes are shown in the original daylight imagery and viewed from the lower of the two altitudes ( 400 and $700 \mathrm{ft}$ ). The images were captured from Microsoft Flight Simulator $98^{\circ}$. In the experiment, the images were rendered in colour. 
(a)
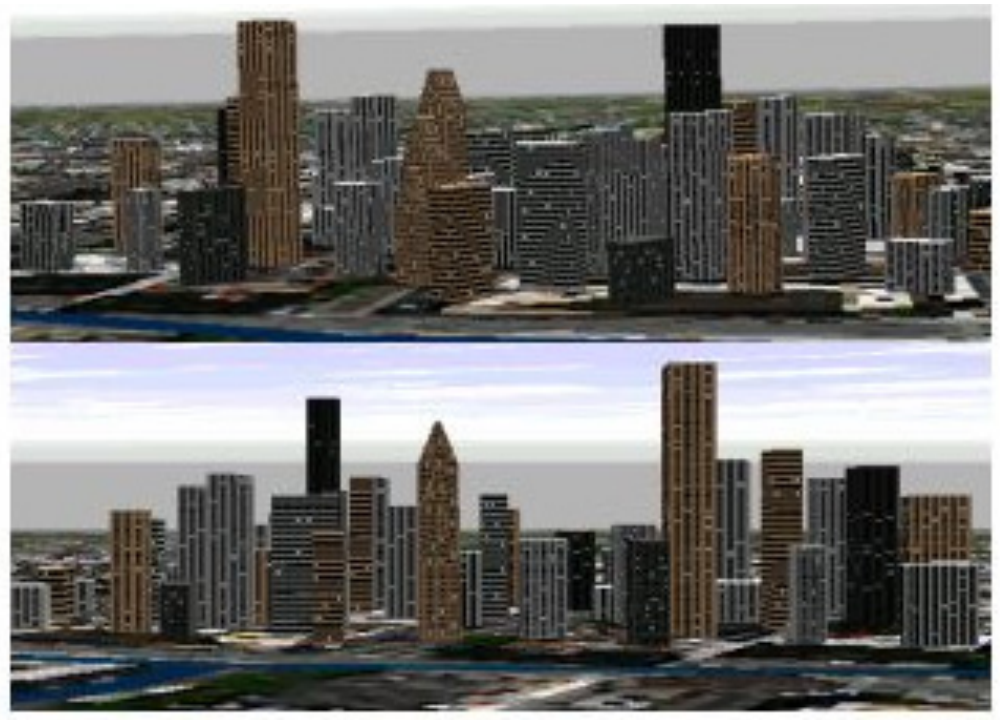

(b)

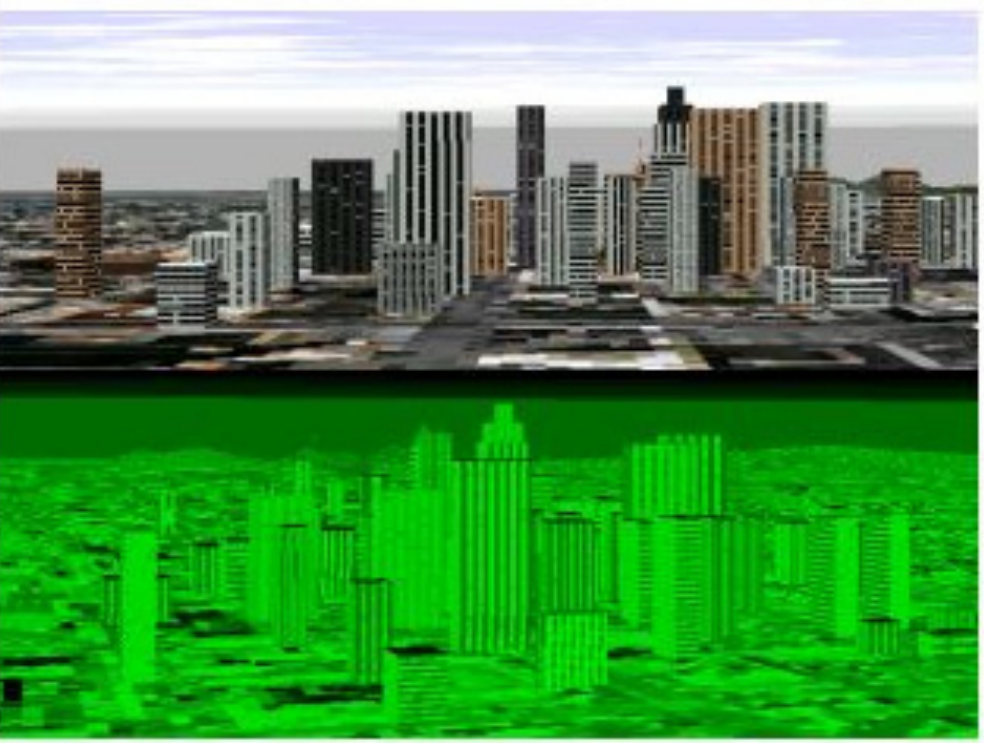

(c)

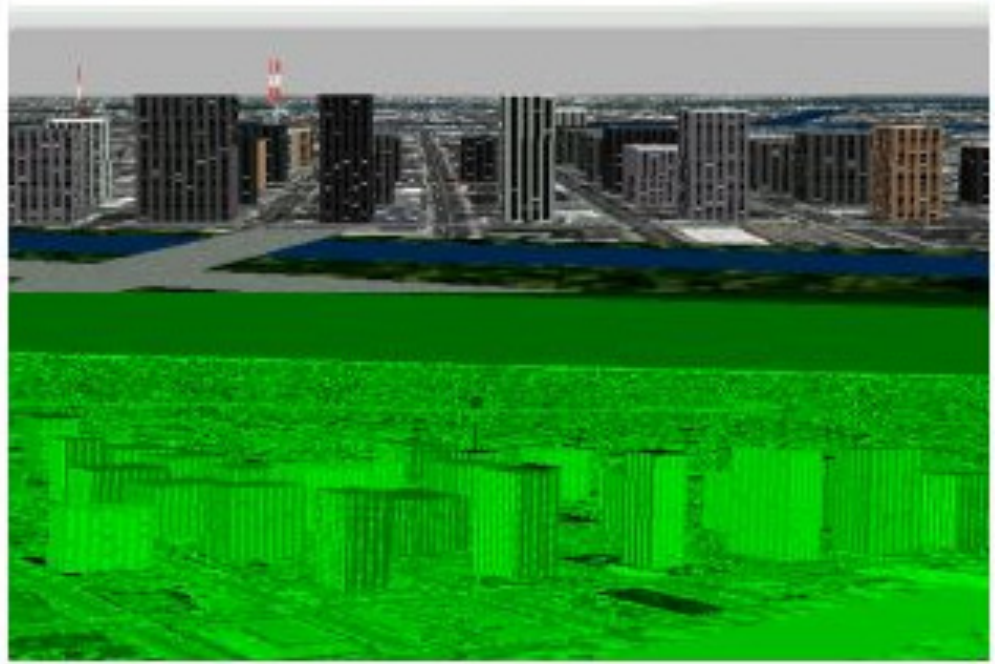

Fig. (3). Example views of some of the stimulus pairs used in the experiment. The effect of the manipulation of reflectances for simulated NVD imagery is also shown. In the experiment, the daylight imagery was rendered in colour, the NVD imagery in green monochrome. 
A Latin square design [17] was used to ensure that each city was used an equal number of times for each of the eight experimental conditions. To achieve this, 16 subjects were used in the experiment. Each subject was assigned to a row in the Latin square. For each subject, there were two trials for each experimental condition, and each of these trials employed one of the 16 different city views. A second Latin square, which was a shifted version of the original (columns were shifted three positions to the right), was used to generate eight practice trials and eight catch trials for each subject, each of which again used a different city. This ensured that no combination of city and condition was repeated during the practice trials or catch trials and the main block of trials and that each city was used only twice within the combined set trials, and never in the identical condition. On the catch trials, the first scene was always a mirror image of the equivalent stimulus used in a non-catch trial. On the practice trials, the second scene was a mirror image of the first was with a 1-in-3 probability. Thus, each subject saw each city only twice during the practice, experimental and catch trials, and on those two occasions the experimental condition was different.

The 8 practice trials were presented as a separate block before the 24 experimental and catch trials. The order of presentation within each block was random.

\subsection{Procedure}

Each participant was given standardised instructions prior to the block of practice trials. The task was explained carefully, in particular the need to discriminate between the rotated and rotated/mirror imaged scenes. The participant's task was to determine whether the difference between the views was due simply to the difference in viewpoint, or if a mirror reversal had also been applied, in which case the two scenes were not a "match". They were alerted to the potential presence of altitude differences in the scenes. The need for correct as well as rapid responses was emphasised, given that navigation requires accurate performance, and we wished to determine how long it took to recognise the scenes under different presentation conditions. The participants were also advised to use an efficient strategy to complete the task. Without this minimal direction, naïve observers sometimes found it very difficult to complete the task. This instruction is reproduced below:

\begin{abstract}
"In order to match the scenes correctly, it is important to use an efficient strategy. To start, identify two buildings that are in both scenes. Then, identify a third building which would define a virtual triangle relative to those two buildings. This triangular configuration of buildings should be present in both scenes, but seen from a different viewing angle. In some scenes, there may be a number of similarlooking buildings, so you should check to eliminate any false matches. If there is a possibility of a false match, try to find a more distinctive building. Keep going until you are confident that the scenes do or do not match".
\end{abstract}

The participants were informed that there was no time limit to their responses. However, if they were still unable to make a decision after approximately two minutes, they were asked to respond as "no match" and to proceed to the next trial. During the practice trials, the experimenter remained in the laboratory, clarified any points raised by the participant, and ensured that the participant clearly understood the task. The experimenter left the laboratory during the main experiment

\subsection{Statistical Analysis}

The data were analysed as a mixed effect model [18] using SPSS version 10.0. Both observer and location were treated as random effects. The viewing altitude of the first and second scenes and the type of imagery (of the second, comparison image) were treated as fixed effects. No examination of the interaction between observer and location was possible due to the use of the Latin Square design, which meant that each subject saw a unique location on each non-catch trial. The primary variable of interest was the type of imagery, but the scene used for those conditions and the individual differences between observers were also examined in the analysis.

\section{RESULTS}

The initial analysis was concerned with the main experimental manipulations of the altitude used to generate the pair of scenes and the imagery used to represent the comparison scene (daylight or NVD). Because there was no evidence of a time-error trade-off, response time for the noncatch trials was the primary variable of interest. The error data will be described below. Mean response times for the eight relevant conditions are shown in Fig. (4). There were no significant interactions involving altitude. Within the overall random effects design, the main effect of imagery was significant, $F(1,15)=13.44, p<.01$. Overall, response times to the simulated NVD scenes were slower, averaging $50.8 \mathrm{~s}$, compared to $34.7 \mathrm{~s}$ for the simulated daylight imagery. This represents a $46 \%$ increase in response time. In addition to this effect, response times were faster if the target scene was viewed from the higher altitude, $F(1,188)=4.44$, $p<.05$. This effect was minor, with responses to image pairs where the target scene was generated from the higher viewpoint $(700 \mathrm{ft}$ ) taking $40.0 \mathrm{~s}$ on average, compared to $45.5 \mathrm{~s}$ when the target scene was viewed from the lower altitude. There were no significant effects involving the viewing altitude of the comparison scene.

The effect of imagery depended on scene characteristics. The average response times to the sixteen different scenes, collapsing across other conditions, are shown in Fig. (5). The main effect of location was significant, $F(15,15)=3.263$, $p<.05$. There was also an interaction between location and type of imagery, $F(15,188)=1.86, p<.05$. Qualitative comparison suggested that the main effect of location was due to the complexity and ambiguity of the scenes, but this was not manipulated quantitatively. The interaction with type of imagery seemed to reflect the degree to which the distinctive colours of individual buildings rendered the scenes less ambiguous in the daylight imagery.

Finally, the effect of the type of imagery on the scene recognition abilities of each participant was examined, averaging across locations. Average response times of the sixteen subjects to daylight and NVD imagery, collapsed 
across the other conditions, are shown in Fig. (6). There was significant variation between observers in their ability to perform the scene recognition task, $F(15,15)=5.846, p<$ .001 , as well as a significant interaction between the observer and the effect of NVD imagery on response time, $F(15,188)=1.905, p<.05$. This showed that these subjectrelated effects were not random, but reflected genuine individual differences in performance.

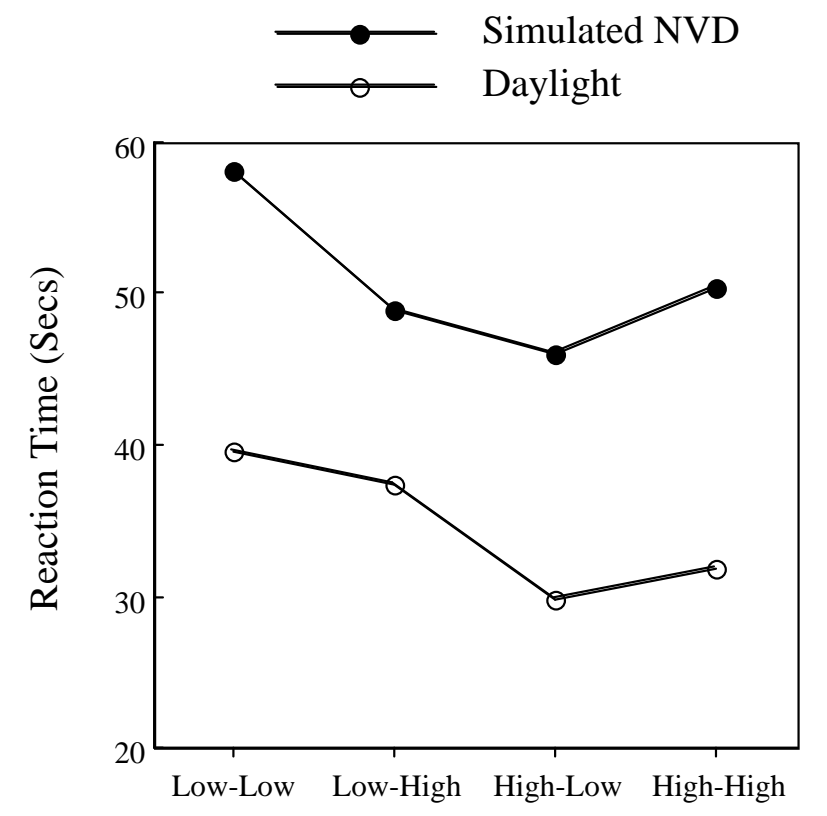

Altitude

Fig. (4). Mean response times for scene recognition as a function of viewpoint altitude and daylight or NVD simulated imagery. Data is averaged over subject/location. Low viewpoints were from $400 \mathrm{ft}$; high viewpoints from $700 \mathrm{ft}$.

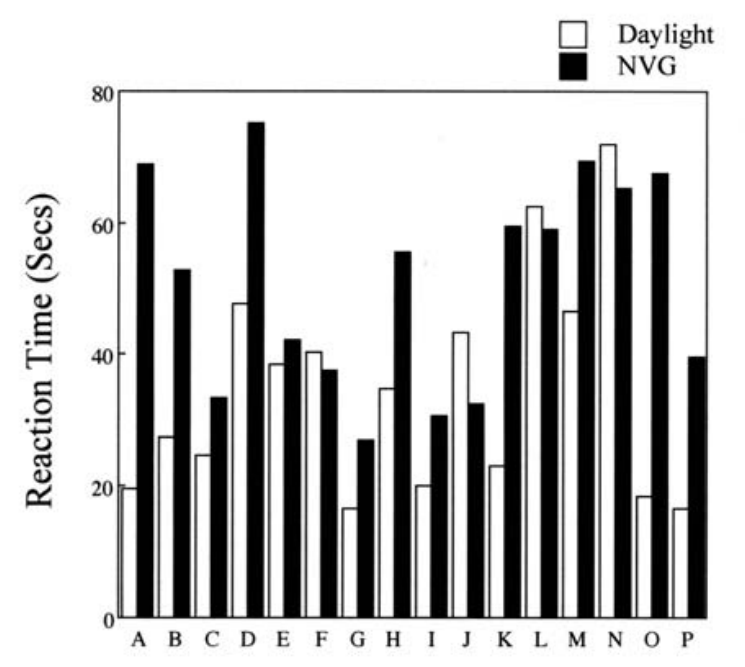

Location

Fig. (5). Mean response times under simulated daylight and NVD simulated conditions for the 16 different locations.

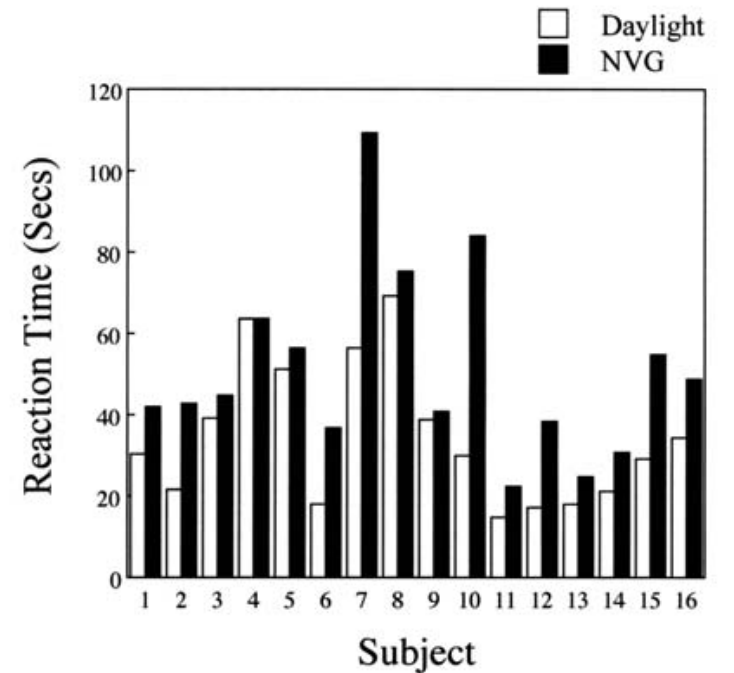

Fig. (6). Mean response times under simulated daylight and NVD simulated conditions for the 16 different subjects.

The analysis of errors showed that they were rather infrequent $(7.03 \%$ of trials), in line with instructions to the participants, and were spread fairly evenly over both locations and the different types of imagery. In total, 13 errors were made on trials using daylight imagery and 15 on trials using NVD imagery. There was no evidence of a speed-accuracy tradeoff, as the error trials were on average slower $(69.5 \mathrm{~s})$ than correct trials $(39.5 \mathrm{~s})$. This indicates that the retention of error trials in the response time analysis biased the results conservatively. The response times would presumably have been even slower had the observer attempted to limit errors even further by using a stricter criterion to ensure correct responses. A reanalysis of the main hypotheses using only the correct trials yielded a very similar pattern of results.

\section{DISCUSSION}

The findings of this study clearly demonstrate that for normal observers, a loss of colour and/or familiar luminance relationships, similar to that associated with NVDs, impairs scene recognition. Both the base level performance and the degree of impairment associated with simulated NVD imagery varied according to specific scene characteristics. In particular, the presence of distinctively coloured landmarks appeared to be important. This is to be contrasted with the findings of Tanaka and Presnell [5] with respect to the recognition of single objects, where colour information benefited performance if it was diagnostic of the object being recognised. In the urban scenes used in the present study, distinctively coloured buildings provide markers of particular locations in the scene. When the scene is rendered in NVD-style imagery it is apparently more difficult to identify corresponding locations when matching the scene. In effect, the observer must ignore the colours and reflectances of the surfaces of the buildings and selectively attend to their size, shape and various details. Different observers also showed significant variation in their ability to carry out this task, but all had problems with at least some scenes. The interpretation of the effects of location and 
observer is complicated by the fact that different observers viewed different sets of locations under simulated NVD and daylight imagery. Nonetheless, despite the variation in performance according to location and observer, a clear deleterious effect of NVD-type imagery, compared with otherwise equivalent colour imagery, was apparent in all conditions. This confirms anecdotal reports of the difficulty of recognising spatial configurations of landmarks when using NVDs.

The use of complex urban scenery produced longer response times than those reported by Hickox and Wickens [15]. They studied the effects of elevation angle, scene complexity and type of feature (built or natural) on the ability to relate an electronic map to the simulated forward view from a cockpit. That is, in contrast to the present study, one view was an exocentric view, provided by the map, and the other the egocentric view from the cockpit. In that study, complexity of the scene was strongly related to the time taken to recognise the scene from the map information. Another difference between the two studies was that in Hickox and Wickens' [15] experiments, only one element was changed in the "same" and "different" conditions. In that study, average response times in the various "same" conditions were under $10 \mathrm{~s}$. In the present study, using daylight imagery, response times in non-catch trials were approximately $35 \mathrm{~s}$ on average, and the use of NVD imagery added a further $15 \mathrm{~s}$ to those response times. Qualitative analysis of the scenes that produced the greatest difficulty in our study suggested that colour played a role in breaking the ambiguity between the scene and its mirror image. That is, in line with basic research, colour diagnosticity was a mediator of performance. Despite some important methodological differences, both studies show that the recognition of specific configurations of objects in a scene reflects a longerterm inspection strategy, rather than an immediate holistic perception. This is to be contrasted with the ability to recognise the general type of scene (e.g., desert, forest, coast) which is very rapid, but which also depends on colour information [6]. It is therefore likely that both aspects of scene recognition will be impaired when using NVDs.

Gauthier et al. [19] reported a recent experiment that complements the findings of the present study. In their study, participants were required to acquire route knowledge and find target objects while navigating a real maze, using unaided vision or when wearing night vision goggles. There are some critical differences between the studies, involving the type of spatial knowledge required to complete the task, and how that knowledge is acquired. There are some important differences between ground-level (route) and aerial view perspectives, and scene recognition is impaired when switching between the two [20]. The use of aerial perspective is more relevant to pilotage using NVDs. The studies also differed in that in the Gauthier et al. [19] study spatial knowledge was acquired through active exploration, whereas in our study it was gained by passive viewing of the scenes. In addition, the use of real night vision goggles confounded the display format with a restricted field of view. Nonetheless, Gauthier et al. [19] found that navigation with NVDs was slower and more error-prone. One possible factor they identified was the associated degradations of visual perception, including loss of colour perception. Our study showed that when all other factors are controlled (including field of view) spatial recognition was impaired by the use of NVD-like rendering of complex urban scenes.

The next generation of NVDs is likely to provide colour imagery. The introduction of colour imagery into NVDs has been shown to improve scene segmentation and target recognition [21]. However, the colours used in these newer devices do not correspond to the natural colours of objects and surfaces, being derived from contrast at infrared and near-infrared/visible wavelengths that is then rendered in false colour. As a result, the overall scene may look less familiar than it does when viewed through monochrome NVDs. The present study showed that the absence of colour information and the unnatural rendering of surface reflectances impaired the recognition of specific urban scenes. Given the deleterious effects of unnatural colouration on the perception of scene gist [6], false colour imagery may impair specific scene recognition even further than monochrome NVDs. Prior to the introduction of colour NVDs, there will be a need to determine to what extent any deleterious effects of unnatural colour on scene recognition outweigh the benefits of colour imagery.

There are a number of limitations to the present study. Navigation is a continuous process, carried out in a dynamic environment. To reach a given destination, a pilot or navigator will plot and follow a course towards it. This ongoing process may provide important information about spatial orientation that will inform decisions at the destination point or along course. In contrast, participants in the present experiment were presented with two static viewpoints on which to base their decision. In addition, motion parallax and other cues in the real world provide important cues to 3-D spatial layout, compared to the more impoverished pictorial depth cues present in the static images used here.

The use of built environments in the experiment means that it is not possible to generalise the results to natural environments, where the available landmarks may be much more ambiguous in character. This suggests that the scene recognition costs of NVD imagery may be even greater in natural environments. Hickox and Wickens [15] found this to be the case when matching map representations to realworld scenes. As the discrepancy in view angle between map and scene increased, the costs for scenes containing "natural" features increased at a greater rate than for those containing "anthropogenic" features. Another factor that may further impair scene recognition ability is the limited field of view of currently available NVDs. This means that only a partial view of the outside scene is available, which may add to the difficulty of scene recognition.

On the basis of these limitations, future experimental studies should employ both natural and built scenes, and both monochrome and colour NVD imagery should be used. If possible, more realistic, dynamic simulation should be used, based on terrain databases that incorporate the correct reflectances at the wavelengths to which the NVDs are sensitive. In addition, it would be useful to use a headslaved, limited field of view aperture to determine any additional costs due to this factor, as in the study of Gauthier et al. [19]. 
There is an increasing emphasis on various kinds of mission rehearsal in which aircrew "fly" a mission in a simulator that recreates the terrain and other elements that will be encountered during the actual mission [22] . Other, less elaborate forms of mission planning and briefing use photographs, maps, drawings or other representations of terrain and relevant features. One of the possible tasks of aircrew during a mission may therefore be to correlate, transform or "rotate" mental images in memory to correspond with actual terrain being encountered in order to maintain geographic situation awareness and stay on track. A question often arises as to the required fidelity of mission rehearsal simulations. Of particular relevance to this study is the necessity of accurate sensor imagery. Does it matter if natural colour daylight imagery is used in a simulator prior to NVD flight, or should simulated NVD imagery be used? The results of the present study suggest there may be benefits to be gained from the latter strategy, but a direct test of this hypothesis is required.

\section{ACKNOWLEDGEMENT}

The authors thank Stephen Westhead for technical assistance during the conduct of this study.

\section{REFERENCES}

[1] Driggers RG, Cox P, Edwards T. Introduction to infrared and electro-optical systems. Boston: Artech House 1999.

[2] Rash CE, Verona RW, Crowley JS. Human factors and safety considerations of night vision systems flight using thermal imaging systems. (USAARL Report No. 90-10). Fort Rucker, AL: United States Army Aeromedical Research Laboratory 1990.

[3] Hughes PK. What is different about vision through night vision goggles? In: Kearney G, Creamer M, Marshall R, Goyne A, Eds. Managing Stress in the Australian Defence Force. Canberra: Defence Publishing Service 2001

[4] Braithwaite MG, Douglass PK, Durnford SJ, Lucas G. The hazard of spatial disorientation during flight using night vision devices. Aviat Space Environ Med 1998; 69: 1038-44.

[5] Tanaka JW, Presnell LM. Colour diagnosticity in object recognition. Percept Psychophys 1999; 61: 1140-53.
[6] Oliva A, Schyns PG. Diagnostic colors mediate scene recognition. Cognit Psychol 2000; 34: 72-107.

[7] Goffeaux V, Jaques C, Mouraux A, Oliva A, Schyns PG, Rossion B. Diagnostic colours contribute to the early stages of scene categorisation: Behavioural and neurophysiological evidence. Vis Cogn 2005; 12: 878-92.

[8] Diwadkar VA, McNamara TP. Viewpoint dependence in scene recognition. Psychol Sci 1997; 8: 302-7.

[9] Nakatani C, Pollatsek A, Johnson SH. Viewpoint-dependent recognition of scenes. Q J Exp Psychol A 2002; 55(A): 115-39.

[10] Friedman A, Waller D. View combination in scene recognition. Mem Cognit 2008; 36: 467-78.

[11] Wickens CD. Frames of reference for navigation. In: Gopher D, Kariat A. Eds. Attention and Performance XVII. Cambridge, MA MIT Press 1999; pp. 113-44.

[12] Eley MG. Determining the shapes of land surfaces from topographic maps. Ergonomics 1988; 31: 355-76.

[13] Aretz AJ, Wickens, CD. The mental rotation of map displays. Hum Perform 1992; 5: 303-28.

[14] Schreiber B, Wickens CD, Alton J, Hickox JC. Navigational checking using 3-D maps: the influence of elevation angle, azimuth and foreshortening. Hum Factors 1998; 40: 209-23.

[15] Hickox JC, Wickens CD. Effects of elevation angle disparity, complexity and feature type in relating out-of-cockpit field of view to an electronic cartographic map. J Exp Psychol Appl 1999; 5: 284-301.

[16] Shepard S, Cooper LA. Mental images and their transformations. Cambridge MA: MIT Press 1982.

[17] Kirk RE. Experimental design: procedures for the behavioral sciences. 2nd ed. Pacific Grove, California: Brooks/Cole Publishing Company 1982.

[18] Winer BJ. Statistical principles in experimental design. 2nd ed. Tokyo: McGraw-Hill Kogakusha, Ltd 1971.

[19] Gauthier MS, Parush A, Macuda T, Tang D, Craig G, Jennings S. The impact of night vision goggles on way-finding performance and the acquisition of spatial knowledge. Hum Factors 2008; 50: 311-21.

[20] Shelton AL, McNamara TP. Orientation and perspective dependence in route and survey learning. J Exp Psychol Learn 2004; 30: 158-70.

[21] Essock EA, Sinai MJ, McCarley JS, Krebs WK, DeFord JK. Perceptual ability with real-world nighttime scenes: Image intensified, infrared and fused-colour imagery. Hum Factors 1999; 41: 438-52.

[22] Bone R, Lintern G. Rehearsal versus map study as preparation for a flight navigation exercise. Hum Factors 1999; 41: 467-73. 